\title{
Bxb1 phage recombinase assists genome engineering in Drosophila melanogaster
}

\author{
Roumen Voutev and Richard S. Mann \\ Departments of Biochemistry and Molecular Biophysics and Systems \\ Biology, Columbia University, New York, NY
}

BioTechniques 62:37-38 (January 2017) doi 10.2144/000114494

Keywords: Bxb1 recombinase; cassette exchange; genome editing; Drosophila melanogaster

Rapid and reliable genome modifications provide the basis for detailed in vivo functional analysis of any genomic entity (gene, regulatory DNA, noncoding RNA, etc). With the advent of CRISPR/Cas9 genome editing technology, manipulation of a particular genomic locus has become a routine undertaking in variety of model organisms, including the fruit fly Drosophila melanogaster. To further diversify the available tools for genome engineering, we successfully harnessed the phage recombinase Bxb1 to perform recombinase-mediated cassette exchange (RMCE) in D. melanogaster. We demonstrate that $\mathrm{Bxb} 1$ possesses highly efficient recombinase activity and could be used alone or in conjunction with other currently available recombinases for creating platforms for cassette exchange of targeted loci.

Site-specific recombinases have revolutionized molecular genetics by allowing researchers to manipulate genomes and gene expression in a highly controlled manner (reviewed in Reference 1). Drosophila melanogaster investigators have adopted and greatly benefited from effective and sustained utilization of a variety of site-specific recombinases of diverse origin, such as Flp (2), Cre (3), and $\Phi C 31$ (4). More recently, several new site-specific recombinase systems have been shown to function in Drosophila, including those based on the Bxb1 (5), KD, B2, B3, and R (6) recombinases, thereby increasing the number of molecular biology tools available for this organism. Here, we present a method utilizing the phage recombinase Bxb1 and its minimal cognate recognition sites (7) to catalyze recombinasemediated cassette exchange (RMCE) of a Bxb1 attP-primed allele using a germline- expressed Bxb1 recombinase. Such an allele could undergo a large number of desired cassette replacements for exhaustive in vivo functional characterization of a locus.

As proof-of-principle for this approach, we inserted a vector bearing the selectable marker hs-neo [heat-shock-protein-70 promoter-driven neomycin resistance gene (neo)] (8) into the fruit fly genome at landing site ZH-51D (9) by ФC31-mediated integration (4) (Figure 1). We designed the vector in such a way that $h s-n e o$ was flanked by inverted minimal Bxb1 attP sites (7) and a loxP site immediately preceding this cassette. Landing site ZH-51D allows for the removal of all intervening vector sequences using Cre/loxPmediated excision (9), which we performed, resulting in a cleaner allele where only the Bxb1 attP-flanked $h s-n e o$ cassette remained at the locus. We named this allele $B B^{\text {hs-neo }}$. Similar Bxb1 attP-primed alleles could be engineered at loci of interest using CRISPR/ Cas9 genome editing technology (10-12).

Next, we generated fly strains that express the phage recombinase Bxb1 in the fly germ line by placing its cDNA under the control of the vasa promoter and the nanos 3' UTR (vasa-Bxb1-nos). We then introduced this construct into landing sites $\mathrm{ZH}-2 \mathrm{~A}(\mathrm{X})$, $\mathrm{ZH}-22 \mathrm{~A}$ (II), and ZH-86Fa (III) (9) through ФC31 transgenesis [the construct contains the selectable marker yellow $(y+)]$. This construct did not exhibit any toxicity since we have maintained the resulting strains for $>3$ years without observable adverse effects.

We also created an RMCE vector that contains a ubiquitin-GFP (ubi-GFP) cassette flanked by inverted minimal Bxb1 attB sites (7) and also contains the selectable marker white $(w+)$ in the vector backbone (Figure 1). We tested if we could efficiently perform RMCE by injection of $250 \mathrm{ng} / \mu \mathrm{l}$ ubi-GFP RMCE vector into embryos resulting from crosses of $B B^{\text {hs-neo }}$ males and vasaBxb1-nos [ZH-2A], vasa-Bxb1-nos[ZH-22A], or vasa-Bxb1-nos[ZH-86Fa] females. Two hundred embryos were injected in each case. We raised the resulting larvae to adulthood at $25^{\circ} \mathrm{C}$. Approximately 15 males and 15 females hatched from each genotype, and we crossed these G0 parents to yw flies.

The Bxb1 recombinase has previously been shown to perform intermolecular recombination in Drosophila (5). Our experimental design allowed us to extend these observations by assessing the ability of Bxb1 to perform RMCE in flies. In addition, we could compare the rate of RMCE with the frequency of recombination events between individual Bxb1 attP and attB sites. Because our ubi-GFP RMCE vector contains the w+ marker in the vector backbone, the latter events would result in vector integration and $w+\mathrm{F} 1$ flies that also fluoresce green as a result of the ubi-GFP cassette. If RMCE occurs, the resulting flies would be ubi-GFP-expressing and $w$-. Interestingly, in the case of vasa-Bxb1-nos[ZH-2A]/4;

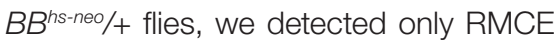
events at a rate of $1.7 \%$ ( $n=600 \mathrm{~F} 1$ flies) and no vector integration events. In the case

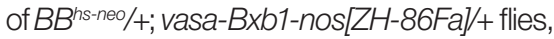
we observed RMCE at a rate of $4.3 \%$ and vector integration events at a rate of $1 \%$ (n

\section{METHOD SUMMARY}

First, a Drosophila melanogaster genomic locus is modified in a way that minimal inverted Bxb1 attP sites flank a region of interest. Next, the phage recombinase Bxb1 is expressed in the fly germ line, where it catalyzes efficient recombinase-mediated cassette exchange (RMCE) between the Bxb1 attP-targeted allele and a donor vector containing cognate minimal inverted Bxb1 attB sites. 
Figure 1. Schematic representation of the ZH-51D landing site locus on chromosome $2 R$ and its genome engineering using Bxb1-catalyzed recombinase-mediated cassette exchange (RMCE). Dark blue represents a $48 \mathrm{bp}$ minimal Bxb1 attP site (GGTTTGTCTGGTCAACCACCGCGGTCTCAGTGGTGTACGGTACAAACC); light blue represents a 38 bp minimal Bxb1 attB site (GGCTTGTCGACGACGGCGGTCTCCGTCGTCAGGATCAT). Plasmid DNA, maps, and complete vector sequences are available from Addgene (Cambridge, MA) at www.addgene.org. Fly strains generated in this study are available from the Bloomington Drosophila Stock Center (Bloomington, IN).

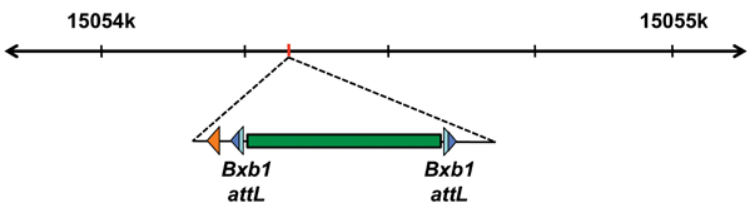

$=600 \mathrm{~F} 1$ flies). These differences could be ascribed either to variations in vasa-Bxb1-nos expression from landing sites $\mathrm{ZH}-2 \mathrm{~A}$ and ZH-86Fa or, possibly, to technical variations in the embryo injections, which often occur between different samples. When each $w+$ F1 fly line, resulting from an integration event, was crossed back to the vasa-Bxb1-nos $[\mathrm{ZH}$ 86Fa] germline integrase strain, intramolecular recombination between the intact attB and attP Bxb1 sites remaining at the locus resulted in completion of the RMCE.

In the previous two experiments, we assessed the rate of Bxb1-driven RMCE as the percentage of GFP-positive F1 flies out of the total number of scored $\mathrm{F} 1$ flies. These rates represent the number of RMCE-transformant flies per unit of injected embryos (200 embryos in this case). In order to also investigate the percentage of independent $\mathrm{G} 0$ flies that yield Bxb1-driven RMCE, we tested a third source of Bxb1 germline integrase: vasa$B \times b 1-n o s[Z H-22 A]$ inserted on the second chromosome. In this case, we found that $18 \%$ (4/22) of G0 BB ${ }^{\text {hs-neo/ } / v a s a-B x b 1-n o s[Z H-~}$ $22 \mathrm{~A}]$ flies injected with the ubi-GFP RMCE vector produced RMCE progeny. We verified successful RMCE events by PCR and sequencing of independent alleles from each genotype. Since the inverted attP/ attB pairs are identical (Figure 1), half of the replaced ubi-GFP cassettes were oriented in the forward orientation and half in reverse, as expected. Overall, we conclude that Bxb1-assisted RMCE in D. melanogaster occurs at rates that are higher than the RMCE rates reported for the Cre/lox system (13) and lower than those reported for the ФC31 system (14).
The successful replacement of $h s-n e o$ with ubi-GFP at the ZH-51D locus using the Bxb1 recombinase for RMCE establishes yet another tool that complements the genome engineering repertoire in the fruit fly. We demonstrated that minimal Bxb1 attP/attB sites, which leave very small DNA scars in the genome, are sufficient for potent RMCE. If positioned carefully, these sites are unlikely to interfere with the function of a particular locus of interest. Moreover, combining Bxb1 with other recombination systems, such as $\Phi$ C 31 , Flp, and Cre, has the potential to add versatility to how genomic loci can be manipulated, not only in $D$. melanogaster but also in other model organisms.

\section{Author contributions}

R.V. conceived this work, performed the experiments, and wrote the article. R.S.M. edited the article and supported the study.

\section{Acknowledgments}

Thanks to David W. Ow for providing us with the Bxb1 cDNA (15); to Rebecca Delker for valuable suggestions; to Lalanti Venkatasubramanian for technical assistance; to Rainbow Transgenic Flies Inc. for fly injections. R.V. is a Leukemia and Lymphoma Society fellow. This work was supported by NIH grant R35GM118336 awarded to R.S.M. This paper is subject to the NIH Public Access Policy.

\section{Competing interests}

The authors declare no competing interests.
References

1. Groth, A.C. and M.P. Calos. 2004. Phage integrases: biology and applications. J. Mol. Biol. 335:667-678.

2. Golic, K.G. and S. Lindquist. 1989. The FLP recombinase of yeast catalyzes site-specific recombination in the Drosophila genome. Cell 59:499-509.

3. Siegal, M.L. and D.L. Hartl. 1996. Transgene Coplacement and high efficiency site-specific recombination with the Cre/loxP system in Drosophila. Genetics 144:715-726.

4. Groth, A.C., M. Fish, R. Nusse, and M.P. Calos. 2004. Construction of transgenic Drosophila by using the site-specific integrase from phage phiC31. Genetics 166:1775-1782.

5. Huang, J., P. Ghosh, G.F. Hatfull, and Y. Hong. 2011. Successive and targeted DNA integrations in the Drosophila genome by Bxb1 and phiC31 integrases. Genetics 189:391-395.

6. Nern, A., B.D. Pfeiffer, K. Svoboda, and G.M. Rubin. 2011. Multiple new site-specific recombinases for use in manipulating animal genomes. Proc. Natl. Acad. Sci. USA 108:14198-14203.

7. Ghosh, P., A.I. Kim, and G.F. Hatfull. 2003. The orientation of mycobacteriophage Bxb1 integration is solely dependent on the central dinucleotide of attP and attB. Mol. Cell 12:1101-1111.

8. Steller, H. and V. Pirrotta. 1985. A transposable $P$ vector that confers selectable G418 resistance to Drosophila larvae. EMBO J. 4:167-171.

9. Bischof, J., R.K. Maeda, M. Hediger, F. Karch, and K. Basler. 2007. An optimized transgenesis system for Drosophila using germ-line-specific phiC31 integrases. Proc. Natl. Acad. Sci. USA 104:3312-3317.

10. Gratz, S.J., A.M. Cummings, J.N. Nguyen, D.C. Hamm, L.K. Donohue, M.M. Harrison, J. Wildonger, and K.M. O'Connor-Giles. 2013. Genome engineering of Drosophila with the CRISPR RNA-guided Cas9 nuclease. Genetics 194:10291035.

11. Kondo, S. and R. Ueda. 2013. Highly improved gene targeting by germline-specific Cas 9 expression in Drosophila. Genetics 195:715-721.

12. Port, F., H.M. Chen, T. Lee, and S.L. Bullock. 2014. Optimized CRISPR/Cas tools for efficient germline and somatic genome engineering in Drosophila. Proc. Natl. Acad. Sci. USA 111:E2967-E2976.

13. Oberstein, A., A. Pare, L. Kaplan, and S. Small. 2005. Site-specific transgenesis by Cre-mediated recombination in Drosophila. Nat. Methods 2:583585.

14. Venken, K.J., K.L. Schulze, N.A. Haelterman, H. Pan, Y. He, M. Evans-Holm, J.W. Carlson, R.W. Levis, et al. 2011. MiMIC: a highly versatile transposon insertion resource for engineering Drosophila melanogaster genes. Nat. Methods 8:737-743.

15. Thomson, J.G. and D.W. Ow. 2006. Site-specific recombination systems for the genetic manipulation of eukaryotic genomes. Genesis 44:465-476.

Received 13 September 2016; accepted 03 November 2016.

Address correspondence to Roumen Voutev or Richard S. Mann, Departments of Biochemistry and Molecular Biophysics and Systems Biology, Columbia University, 701 W168th St. HHSC 1104, New York, NY 10032. E-mail: roumen.voutev@gmail.com or rsmann10@gmail.com

To purchase reprints of this article, contact: biotechniques@fosterprinting.com 